\title{
THE PREPARATION OF DIETHYL MALONIC ESTER.
}

\author{
By W. A. Noyes. \\ Received October 29, 1896.
}

AVING occasion recently to prepare considerable quantiI ties of malonic ester, it has been found that the process can be very much shortened by the use of sulphuric in place of hydrochloric acid and of acid sodium carbonate in place of potassium carbonate. As the body is the starting point for a great variety of syntheses the method used may be of interest to others.

One hundred grams of chloracetic acid are placed in a porcelain dish, $2 \mathrm{I} \mathrm{cm}$. in diameter, and $200 \mathrm{cc}$. of water added. The solution is warmed and ninety grams of acid sodium carbonate added in small portions, and the warming continued until a temperature of $55^{\circ}-60^{\circ}$ is reached and effervescence nearly ceases. Eighty grams of coarsely powdered potassium cyanide is then added, and the whole stirred without further warming, till the somewhat vigorous reaction is complete. The solution is then evaporated rapidly on a thin sheet of asbestos paper till the thermometer with which it is vigorously and constantly stirred shows a temperature of $130^{\circ}-135^{\circ}$. The hand should be protected by a glove or otherwise, and the glass of the hood in which the evaporation is conducted should be between the dish and the face during this part of the process. The mass should be stirred occasionally while cooling, and as soon as it solidifies it should be broken up coarsely and transferred to a liter flask. Add forty cc. of alcohol and connect with an upright condenser. Through the latter add, in small portions and with frequent shaking, a cooled mixture of $\mathrm{I} 60 \mathrm{cc}$. of alcohol with I6o cc. of concentrated sulphuric acid. The whole may be added within five or ten minutes (instead of the day and a half required to saturate with hydrochloric acid by the old method). Toward the close there is a considerable evolution of hydrochloric acid. Heat on a water-bath for an hour. Cool quickly under the tap, with shaking to prevent the formation of a solid mass of crystals. Add $200 \mathrm{cc}$. of water, filter, wash the undissolved salts with about fifty cc. of ether, shake up with the filtrate and separate. Add a solution of sodium carbonate and shake carefully 
with the ethereal solution till alkaline. Separate again, distil off the ether and dry by heating for fifteen minutes on a waterbath under diminished pressure, using a capillary tube as for vacuum distillations. The residue gives, after one distillation, an almost pure malonic ester.

The sodium carbonate solution appears to contain some of the acid ester. If this solution is added to the first acid solution, the ester separates with some ether. The ethereal solution may be separated, the ether evaporated at a gentle heat, and the residue added to the contents of the flask in which a second saponification of the cyanacetate is to be effected. If this is done, a yield of malonic ester equal to the weight of chloracetic acid used can be obtained. This is ten to fifteen per cent. better than by the old nethod.

Rose POLYTEChNic Institute,

TERRE HAUTE, IND., Oct. 27, I896.

\section{NOTE.}

Untaxed Alcohol for Use in Manufacturing and in the Arts.The Joint Select Committee, created at the last session of Congress, to investigate and report upon the question of the use of alcohol free of $\operatorname{tax}$ in the manufactures and arts, have prepared a series of interrogatories, which will be distributed throughout the country to such parties as are thought to be interested in the question.

The report of Mr. Henry Dalley, Jr., who was commissioned to investigate the workings of foreign laws governing the use of untaxed alcohol in the manufactures and arts has been submitted, and contains very full and extremely valuable data covering Great Britain, Germany, France, Belgium and Switzerland.

It is the earnest desire of the committee to secure all possible information bearing upon the subject, and it is hoped that parties interested will submit their views to the committee promptly. Sets of the circular letter and blank for replies will be supplied to any applicant by addressing the chairman, Room 2 I, Senate Annex, Washington, D. C.

The committee, which is composed of three members of each House, will probably assemble in Washington soon after the 